\title{
Asociación de la competencia en las habilidades motrices básicas con las actividades físico- deportivas extracurriculares y el índice de masa corporal en preescolares Association of the fundamental movement skills competence with the extracurricular sports and the body mass index in preschoolers
}

Pablo García-Marín, Natalia Fernández-López

Universidad de Santiago de Compostela (España)

Resumen: El objetivo del estudio fue analizar la competencia en las habilidades motrices básicas de preescolares gallegos y su grado de asociación con las actividades físico-deportivas extracurriculares y el IMC. Se utilizó un diseño ex post-facto descriptivo correlacional.

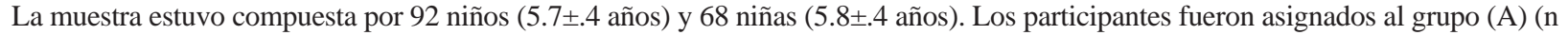
= 92) cuando realizaron un mínimo de dos horas a la semana de actividades físico-deportivas extracurriculares durante los últimos seis meses, o al grupo (B) $(n=68)$, si no cumplían con el requisito anterior. Las habilidades motrices fueron evaluadas con el Test of Gross Motor Development-2 $2^{a}$ edition. El grupo (A) alcanzó mayor puntuación en el coeficiente motor grueso $(p<.001)$, en la escala de habilidades locomotrices ( $p<.001$ ), en la de control de objetos $(p<.001)$ y en todas las habilidades, excluyendo la de galopar $(p=.294)$. El IMC se asoció con la competencia motriz $(p<.001)$ y con la participación en actividades físico-deportivas extracurriculares $(p=.001)$. Además, correlacionó con el coeficiente motor grueso $(p=.009)$, la escala de habilidades locomotrices $(p=.017)$ y la de control de objetos $(p=.018)$, así como con galopar $(p<.001)$, saltar un obstáculo $(p=.001)$ y chutar $(p=.045)$. La práctica de actividades físico-deportivas extracurriculares se asoció con mejores niveles de competencia motriz y menores índices de sobrepeso y obesidad en los preescolares. Palabras clave: Competencia motriz; habilidades motrices básicas; deporte extracurricular; índice de masa corporal; preescolares.

Abstract: The aim of the study was to analyze the fundamental movement skills competence of the Galician preschoolers and its association with the extracurricular sports and the BMI. A correlational descriptive ex post facto design was used. The sample was

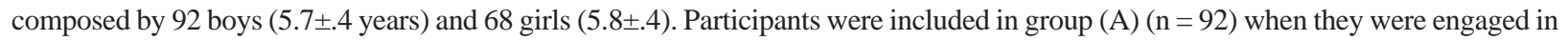
extracurricular sports a minimum of two hours/week over the past six months, or in group (B) (n=68), if they did not meet the previous condition. The fundamental movement skills were assessed with the Test of Gross Motor Development-2 ${ }^{a}$ edition. Group (A) reached significatively more score in the gross motor quotient $(p<.001)$, the locomotor scale $(p<.001)$, the object control scale $(p<.001)$ and in all the fundamental movement skills, excluding gallop $(p=.294)$. BMI was associated with motor competence $(p<.001)$ and extracurricular sports $(p=.001)$. In addition, it was correlated with the gross motor quotient $(p=.009)$, the locomotor scale $(p=.017)$, the object control scale $(p=.018)$, as well as with gallop $(p<.001)$, leap $(p=.001)$ and kick ( $p=.045)$. The practice of extracurricular sports was associated with better levels of motor competence and lower rates of overweight and obesity in the preschoolers.

Keywords: Motor competence; fundamental movement skills; extracurricular sports; body mass index; preschoolers.

\section{Introducción}

Las habilidades motrices básicas (HMB) constituyen el alfabeto de la motricidad (Batalla, 2000; Ureña, Ureña \& Alarcón, 2008), la base sobre la que se construyen las respuestas motrices más complejas (Gallahue, Ozmun \& Goodway, 2011). Suelen involucrar a dos o más segmentos corporales (Gallahue \& Donelly, 2003) y pueden clasificarse en locomotrices, manipulativas o de control/manejo de objetos y de equilibrio (Gallahue et al., 2011).

El aprendizaje y la adquisición de las HMB se produce progresivamente entre los cuatro y los diez años (Gallahue et al., 2011) en paralelo a la maduración del sistema nervioso (Malina, Bouchard \& Bar-Or, 2004). Por este motivo, la etapa preescolar es clave para promover su desarrollo y deben ser tenidas en cuenta en el ámbito educativo. Estudios anteriores han identificado varios factores que promueven su aprendizaje, como por ejemplo: las características del entorno y del equipamiento, que debe ser amplio y atractivo (Barnett, Hinkley, Okely \& Salmon, 2013); la formación y competencia de los docentes para planificar los procesos de enseñanzaaprendizaje, dar instrucciones y motivar la participación del alumnado (Adamo et al., 2016); la implicación de las familias

Fecha recepción: 03-04-19. Fecha de aceptación: 02-11-19

Pablo Garcia-Marin

pablo.garcia@usc.es y la colaboración del entorno social para apoyar actividades de ocio que conlleven la experimentación motriz (Cools, De Martelaer, Samaey \& Andries, 2011); o la implementación de programas específicos como el Multimove for Kids (Bardid et al., 2017), o el Children's Health Activity Motor Program (Veldman, Palmer, Okely \& Robinson, 2017). Por el contrario, se ha evidenciado que la participación de los preescolares en juegos de elección libre, en comparación con los programas realizados en entornos enriquecidos y dirigidos por especialistas, no consigue los mismos niveles de competencia motriz (Robinson, Palmer \& Bub, 2016; Stocke, Oliveira \& Cristina, 2014). En síntesis, los estímulos y el entorno deben suponer una continua exigencia de adaptación donde los niños tengan que ejercitarse motrizmente y emplear simultáneamente sus habilidades cognitivas, sociales y afectivas para comprender el mundo que les rodea, crecer y ser más autónomos.

El modelo de Stodden et al. (2008) establece que la competencia en las HMB durante la primaria infancia determina la percepción subjetiva de la misma (LeGear et al., 2102), la participación en actividades físicas (Logan, Webster, Getchell, Pfeiffer \& Robinson, 2015; Lubans, Morgan, Cliff, Barnett \& Okely, 2010), el nivel de condición física (Catuzzo et al., 2016) y la composición corporal (Barnett et al., 2016). De esta manera, los infantes con buena competencia en las HMB tendrían una percepción positiva que los conduciría a ser más activos y a mejorar su condición física, disminuyen- 
do así el riesgo de padecer obesidad o sobrepeso. A la inversa ocurriría con los menos competentes a nivel motor. En consecuencia, el dominio de las HMB en edades tempranas se ha vinculado con una actividad física placentera y con un estilo de vida saludable, hábitos que se mantendrían también en las siguientes etapas de la vida (Lubans et al., 2010).

Además de los beneficios comentados anteriormente, la importancia de adquirir un buen desarrollo de las HMB en la etapa preescolar radica en la influencia positiva que tienen en otras habilidades cognitivas, sociales y afectivas. Así, los niños con mejor competencia motriz tendrían mayores capacidades para leer y escribir (Callcot, Hammond \& Hill, 2015), para inhibirse (Wu, Lian, Lu \& Wang, 2017) o para controlar voluntariamente la atención, las emociones y los impulsos (Robinson et al., 2016). Todas estas habilidades han sido identificadas como factores de éxito académico, destacando el papel de la motricidad como mediador en el rendimiento escolar (Becker, McClelland, Loprinzi \& Trost, 2014).

Los antecedentes a este trabajo encontraron coeficientes motores gruesos inferiores a los que corresponderían por edad en 267 preescolares canadienses ( $5 \pm .9$ años) (LeGear et al., 2012) y 284 brasileños (3-6 años) (Spessato, Gabbard, Valentini \& Rudisill, 2012). En el contexto español, el nivel de logro alcanzado por 70 asturianos/as (4-6 años) también fue pobre, obteniendo un percentil 37 en las habilidades locomotrices y un 5 en las de control de objetos (González, Cecchini, López \& Riaño, 2009).

Estos resultados llevan a cuestionarse el tratamiento de la educación física en las escuelas infantiles para proporcionar una adecuada competencia motriz. Aunque en la población preescolar faltan estudios al respecto, la investigación de Pons \& Arufe (2016) reveló algunas carencias que podrían limitar el desarrollo motor del alumnado en las escuelas gallegas. En concreto, se evidenció que el 18\% de las escuelas infantiles no incluían sesiones de motricidad en su programación y que el 30\% de las clases de educación física se realizaban en espacios no diseñados para la actividad física (aulas de clase, usos múltiples, salón de actos, etc.). Además, el promedio de tiempo dedicado a las sesiones de motricidad fue de 63 minutos a la semana, tiempo que parece escaso si se tienen en cuenta las tres horas al día de actividad física a diferentes niveles de intensidad que se recomiendan para la población infantil (Piercy et al., 2018). En esta línea Padial-Ruz, Ibáñez-Granados, Fernández-Hervas \& Ubago-Jiménez (2019) señalaron que los docentes, a pesar de reconocer la importancia de la motricidad en la etapa preescolar, le dedican menos tiempo que al aprendizaje de otras habilidades (lógico-matemáticas, lectura, escritura o inglés) al no sentirse suficientemente preparados para abordar su enseñanza-aprendizaje.

Por otra parte, la oferta de actividades físico-deportivas fuera del horario escolar es amplia y variada. Actualmente, los preescolares tienen muchas oportunidades de practicar todo tipo de deportes (patinaje, natación, balonmano, pádel, fútbol, judo...) en escuelas públicas de iniciación deportiva, clubes privados u otros tipos de asociaciones. Gracias a ello entre el 58\% y el 69\% de los preescolares realizan actividad físico-deportiva extracurricular entre dos y tres horas a la semana (Delgado-Lobete \& Montes-Montes, 2015; Delgado-Lobete \& Montes-Montes, 2017). Los deportes ofrecen una alternativa para activarse físicamente, así como para favorecer el desarrollo psicosocial, la cooperación, la disciplina, el liderazgo y la autorregulación (Malina, 2009). Además, constituyen una vía para incrementar el tiempo de actividad física de intensidad moderada y vigorosa (Wickel \& Eisenmann, 2007), y tienen el potencial de prevenir problemas cardiovasculares y de obesidad en niños (Malina, 2009). Asimismo, la práctica deportiva se ha asociado positivamente con el rendimiento escolar (Bekchechi \& Khiat, 2019). No obstante, hay que señalar que existen contextos de especialización deportiva temprana que pueden restringir la competencia motriz de los preescolares al emplear excesivamente las habilidades específicas en detrimento de las básicas (Šalaj, Krmpotiæ \& Stamenkoviæ, 2016).

Dada la importancia de las HMB en la etapa preescolar como precursoras de un estilo de vida físicamente activo y saludable, se plantea este estudio con el objetivo de conocer la competencia en las HMB de los preescolares gallegos de cinco años y su asociación con las actividades físico-deportivas extracurriculares y el índice de masa corporal (IMC).

\section{Material y método}

\section{Diseño y muestra}

El diseño de la investigación fue del tipo ex post-facto descriptivo correlacional. Se empleó un muestreo no probabilístico accidental. La muestra estuvo compuesta por 160 niños y niñas de seis escuelas infantiles públicas gallegas. Todos los participantes se clasificaron en dos grupos (A) y (B). El criterio de inclusión para pertenecer al grupo (A) fue realizar un mínimo de 2 horas a la semana de actividades físico-deportivas extracurriculares durante los seis meses anteriores a la investigación. Para comprobarlo, se consultó a las familias de los participantes. Todos los que no cumplieron con el criterio de inclusión fueron asignados al grupo (B), cuya actividad física organizada se circunscribía exclusivamente al ámbito escolar. Finalmente, 92 participantes de los que completaron la evaluación de las HMB pertenecieron al grupo (A) (57.5\%) y 68 al (B) (42.5\%) (Tabla 1).

\begin{tabular}{|c|c|c|c|c|c|c|}
\hline & & n (\%) & Edad (años) & $\operatorname{Pes}(\mathrm{kg})$ & Fstatura $(\mathrm{cm})$ & $\mathrm{JMC}\left(\mathrm{kg} \cdot \mathrm{m}^{-2}\right)$ \\
\hline \multirow{3}{*}{ (A) } & Niños & 60 (37.5\%) & $5.7 \pm .3$ & $22.9 \pm 2.0$ & $119.9 \pm 4.5$ & $15.9 \pm 0.9$ \\
\hline & Niñas & $32(20.0 \%)$ & $5.7 \pm .4$ & $21.0 \pm 2.3$ & $116.0 \pm 3.1$ & $15.5 \pm 1.0$ \\
\hline & Total & 92 (57.5\%) & $5.7 \pm .4$ & $22.3 \pm 2.3$ & $118.6 \pm 4.5$ & $15.8 \pm 1.0$ \\
\hline \multirow[t]{3}{*}{ (B) } & Niños & $32(20.0 \%)$ & $5.7 \pm .4$ & $24.0 \pm 5.2$ & $115.8 \pm 8.4$ & $17.8 \pm 2.6$ \\
\hline & Niñas & $36(22.5 \%)$ & $5.8 \pm .3$ & $22.8 \pm 3.7$ & $118.1 \pm 2.4$ & $16.3 \pm 2.0$ \\
\hline & Total & 68 (42.5\%) & $5.8 \pm .4$ & $23.4 \pm 4.5$ & $117.1 \pm 6.0$ & $17.0 \pm 2.4$ \\
\hline
\end{tabular}

La edad $\left(\mathrm{F}_{1,158}=1.467 ; p=.228\right)$, el peso $\left(\mathrm{F}_{1,158}=3.476 ; p=\right.$ $.065)$ y la talla $\left(\mathrm{F}_{1,158}=3.219 ; p=.075\right)$ fueron similares para ambos grupos. El IMC difirió significativamente $\left(\mathrm{F}_{1,158}=\right.$ 14.441; $<$.001).

\section{Antropometría}

Las medidas antropométricas se tomaron con ropa ligera y sin zapatos antes de realizar la evaluación de las HMB. El peso se midió con una báscula Tanita UM-804 y la talla con un estadiómetro ADE MZ10042. Con ambas medidas se calculó el índice de masa corporal (IMC = masa [kg] • estatura $\left.\left[\mathrm{m}^{-2}\right]\right)$, considerado el indicador antropométrico más práctico, accesible, universal, barato y no invasivo para clasificar el sobrepeso y la obesidad (de Onis \& Lobstein, 2010). Para 
tal fin, se tomó como referencia los patrones de crecimiento de la OMS. Se consideró sobrepeso cuando se superó una desviación típica el IMC correspondiente a la edad, y obesidad cuando se sobrepasaron dos desviaciones típicas (de Onis \& Lobstein, 2010).

\section{Evaluación de las habilidades motrices básicas}

Se utilizó el Test of Gross Motor Development-2 ${ }^{a}$ edition (TGMD-2) (Ulrich, 2000). Este instrumento valora seis habilidades locomotrices (HL): correr, galopar, saltar a la pata coja, saltar un obstáculo con carrera previa, saltar horizontalmente desde parado y deslizarse lateralmente sobre una línea sin desviarse; y otras seis de control de objetos (HCO): batear una pelota que está en estático, botar un balón de baloncesto con la mano dominante cuatro veces, recepcionar una pelota con las dos manos, chutar un balón que está estático, lanzar una pelota por encima de la cabeza con la mano dominante y lanzar rodando una pelota entre dos conos con la mano dominante.

Cada habilidad se descompone en tres o cuatro criterios de rendimiento cualitativo referidos al patrón motor y que constituyen el objeto de la evaluación. El test proporciona una puntuación del coeficiente motor grueso derivado de la competencia demostrada en el conjunto de las doce HMB, una puntuación estándar de la escala de HL y otra de HCO. Además, estima la edad cronológica teórica del desarrollo motor y los percentiles de cada escala (HL y HCO), así como del conjunto de las habilidades motrices.

Para administrar el test es necesario el siguiente material: un balón de 20 a $25 \mathrm{~cm}$, una pelota de $10 \mathrm{~cm}$, un balón de baloncesto, una pelota de tenis, un balón de fútbol, una pelota de softball, un saquito de 10 a $12 \mathrm{~cm}$, dos conos, un tee de bateo y cinta adhesiva.

La fiabilidad del instrumento (á de Cronbach) es de 0.91 para el coeficiente motor grueso, de 0.85 en las HL y de 0.88 en las HCO. El manual del examinador garantiza la validez de contenido, predicción y de constructo del TGMD-2 (Ulrich, 2000).

\section{Procedimiento}

Se solicitó autorización a los centros escolares y a los docentes implicados para tomar las medidas antropométricas y administrar el TGMD-2. También se requirió el consentimiento informado a las familias del alumnado, en el que se especificaron las pruebas a realizar, el protocolo a seguir y el carácter voluntario de la participación. Con el fin de poder revisar los resultados, también se solicitó autorización para filmar con videocámara. A todos los participantes se les asignó un código y se les garantizó el tratamiento de sus datos de forma anónima y confidencial.

Dos examinadores evaluaron las HMB siguiendo las instrucciones del manual (Ulrich, 2000). Previamente, se realizaron dos sesiones de entrenamiento en las que evaluaron a diez niños distintos de la muestra final. Posteriormente, se calculó la concordancia inter e intraexaminador con el coeficiente Kappa evaluando a otros diez preescolares en dos ocasiones con una diferencia de dos semanas. La concordancia interexaminador final fue de .84 y la intraexaminador de .86 .

Para realizar el test los participantes fueron llamados in- dividualmente en un espacio tranquilo. Primero, uno de los examinadores describía verbalmente la habilidad y realizaba una demostración práctica. A continuación se facilitaba un tiempo de prueba y, finalmente, se procedía con el registro de los resultados.

Los participantes realizaron dos intentos de cada HMB. Los examinadores asignaron 1 punto cuando se demostró competencia en el criterio de rendimiento y 0 puntos cuando la ejecución era incorrecta. Todo el alumnado fue evaluado por los dos examinadores simultáneamente que consensuaron el registro. En caso de duda, los examinadores revisaron los resultados con las imágenes filmadas. Cada participante invirtió un tiempo aproximado de 15 a 20 minutos en completar el test.

\section{Análisis Estadístico}

Se calcularon las medias y desviaciones típicas de las variables (coeficiente motor grueso, escala de HL, de HCO y cada una de las HMB) para los grupos (A) y (B) de forma independiente. Al no cumplir el supuesto de normalidad comprobado con la prueba de Kolgomorov-Smirnov, se realizó un análisis comparativo entre ambos grupos empleando la prueba U de Mann-Whitney. Se calculó el tamaño del efecto mediante $\mathrm{r}=\mathrm{Z} / \sqrt{\mathrm{n}}$, considerándose trivial cuando $\mathrm{r}<.1$, pequeño .1-.3, medio .3-.5 y grande $>$.5.

Se utilizó la prueba Chi-cuadrado para comprobar la existencia de dependencia entre la práctica de actividades físicodeportivas extracurriculares y los niveles de competencia motriz en el TGMD-2 utilizando los valores normalizados de Ulrich (2000). También para comprobar la asociación con el IMC, que se clasificó en delgadez severa, delgadez, normalidad, sobrepeso y obesidad utilizando los puntos de corte propuestos por la OMS. Las intensidades de las asociaciones en ambos casos se estimaron con la V de Cramer.

Además, se calcularon las correlaciones entre el IMC y las puntuaciones estándar del coeficiente motor grueso, las escalas de HL y de HCO, y cada una de las HMB.

El nivel de significación asumido en todas las pruebas fue de $p \leq .05$. Todos los análisis estadísticos se ejecutaron con el SPSS software package, versión 20.0 (SPSS Inc., Chicago, IL, USA).

\section{Resultados}

El coeficiente motor grueso alcanzó diferencias significativas en función de la participación en actividades físicodeportivas extracurriculares ( $\mathrm{Z}=-8.428 ; p<.001 ; \mathrm{TE}=.67)$. El alumnado implicado en dichas actividades (A) obtuvo una puntuación de $100 \pm 12.2$, mientras que el que no lo hizo (B)

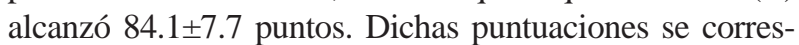
pondieron con un percentil de $52.1 \pm 22.4$ y de $17.2 \pm 13.8$ respectivamente.En la Figura 1 se muestran los niveles de competencia en las HMB para ambos grupos tomando como referencia las edades cronológicas teóricas de desarrollo (Ulrich, 2000).

Así, el 87\% del grupo (A) alcanzó o superó la competencia motriz correspondiente a su edad. Por el contrario, el $70.6 \%$ del grupo (B) obtuvo resultados por debajo de la misma. Se encontraron diferencias significativas entre los dos $\operatorname{grupos}\left(\chi^{2}=58.361 ; p<.001 ; \mathrm{V}=.604\right)$. 


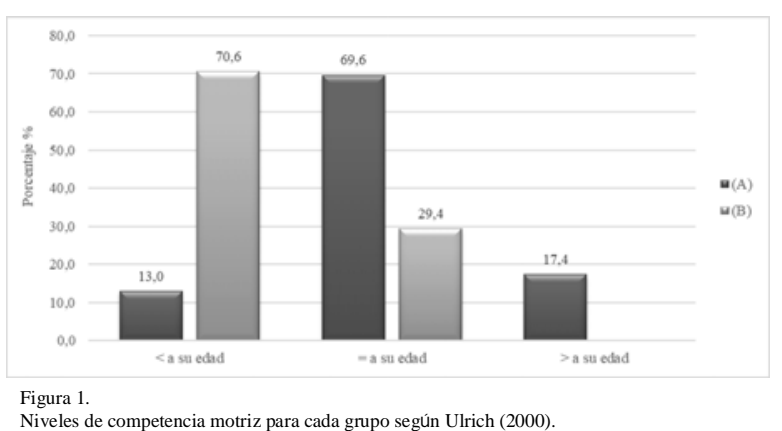

Las puntuaciones estándar alcanzadas en las escalas de $\mathrm{HL}(\mathrm{Z}=-7.633 ; p<.001 ; \mathrm{TE}=.60) \mathrm{y}$ de HCO $(\mathrm{Z}=-7.790 ; p<$ .001 ; $\mathrm{TE}=.62$ ) también difirieron significativamente para ambos grupos, observando puntuaciones más altas en el grupo (A) en todos los casos (Tabla 2).

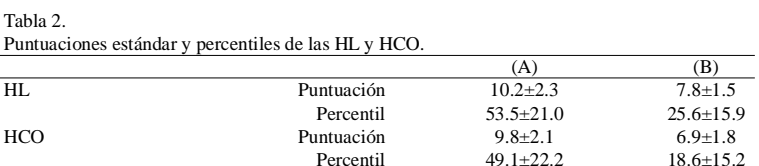

Mientras que en el grupo (A) las puntuaciones estándar de las HL y de HCO fueron similares ( $\mathrm{Z}=-1.589, p=.112)$, en el grupo (B) las de las HL fueron significativamente superiores a las de $\mathrm{HCO}(\mathrm{Z}=-3.406 ; p=.001 ; \mathrm{TE}=.27)$.

Las puntuaciones obtenidas en cada $\mathrm{HMB}$ se muestran en la Tabla 3. En todas ellas el grupo (A) obtuvo más puntuación que el (B), alcanzando diferencias significativas excepto en galopar $(Z=-1.049 ; p=.294)$. Los tamaños del efecto fueron pequeños: deslizar (.22) y lanzar rodando (.29); y medios: pata coja (.31), batear (.34), botar (.35), saltar obstáculo (.41), lanzar (.45), recepcionar y chutar (.46), salto horizontal (.49) y correr (.50).

\begin{tabular}{|c|c|c|c|c|c|}
\hline \multicolumn{6}{|l|}{$\overline{\mathrm{HMB}}$} \\
\hline \multirow[t]{6}{*}{ HL } & Correr & $6.43 \pm 1.4$ & $4.47 \pm 2.0$ & -6.379 & $<.001$ \\
\hline & Galopar & $6.22 \pm .2$ & $5.88 \pm 1.4$ & -1.049 & .294 \\
\hline & Pata Coja & $6.30 \pm 2.1$ & $5.29 \pm .6$ & -3.934 & $<.001$ \\
\hline & Saltar obstáculo & $4.43 \pm .4$ & $3.29 \pm .4$ & -5.154 & $<.001$ \\
\hline & Salto horizontal & $5.78 \pm 1.4$ & $4.06 \pm .5$ & -6.231 & $<.001$ \\
\hline & Deslizar & $6.70 \pm 2.0$ & $6.47 \pm .2$ & -2.825 & .005 \\
\hline \multirow[t]{6}{*}{$\mathrm{HCO}$} & Batear & $6.30 \pm .9$ & $4.82 \pm 2.2$ & -4.239 & $<.001$ \\
\hline & Botar & $4.48 \pm .9$ & $2.71 \pm 2.0$ & -4.411 & $<.001$ \\
\hline & Recepcionar & $4.83 \pm .1$ & $3.53 \pm .3$ & -5.759 & $<.001$ \\
\hline & Chutar & $5.70 \pm .6$ & $4.18 \pm .3$ & -5.763 & $<.001$ \\
\hline & Lanzar & $4.65 \pm 2.0$ & $3.29 \pm .0$ & -5.712 & $<.001$ \\
\hline & Lanzar rodando & $5.61 \pm .6$ & $4.59 \boxplus .1$ & -3.722 & $<.001$ \\
\hline
\end{tabular}

El IMC y la participación en actividades físico-deportivas extracurriculares mostraron una asociación de dependencia $\left(X^{2}=11.101 ; p=.001 ; \mathrm{V}=.263\right)$. En el grupo (B) el $35.3 \%$ del alumnado presentó sobrepeso u obesidad, apareciendo 3.3 más casos de los que cabría esperar, en contraste con el grupo (A), cuyo porcentaje de sobrepeso y obesidad solo alcanzó el $13 \%$.

En el conjunto de la muestra, el IMC correlacionó significativamente con el coeficiente motor grueso $(\mathrm{r}=-.206$; $p=.009)$, así como con las HL ( $\mathrm{r}=-.189 ; p=.017)$ y las de HCO ( $\mathrm{r}=-.187 ; p=.018)$. De forma específica también correlacionó con galopar $(\mathrm{r}=-.297 ; p<.001)$, saltar un obstáculo $(\mathrm{r}=-.267 ; p=.001)$ y chutar $(\mathrm{r}=-.158 ; p=.045)$.

\section{Discusión}

El objetivo de este estudio fue analizar la asociación de la competencia en las HMB con la práctica de actividades físico-deportivas extracurriculares y el IMC en niños y niñas de cinco años pertenecientes a escuelas infantiles gallegas. En relación con éste, se comprobó que la práctica deportiva realizada con una frecuencia mínima de dos horas a la semana durante seis meses, dirigida por un técnico y realizadas en horario extraescolar se asoció positivamente con la competencia en las HMB. Así lo confirmaron los resultados relativos al coeficiente motor grueso, las escalas del test (HL y HCO) y todas las HMB, mejores para el grupo de preescolares que practicó deporte extracurricular. La única habilidad motriz que no siguió esta tendencia fue la de galopar.

Dichos resultados coinciden con los de otras investigaciones que, de forma general, también encontraron asociación entre ambas variables. Concretamente, Queiroz, Ré, Henrique, Moura \& Catuzzo (2014), en una muestra de preescolares brasileños de tres a cinco años, encontraron puntuaciones más altas en la escala de HL, en la de HCO y en la habilidad de deslizar en el grupo que practicaba deporte extracurricular dos horas a la semana frente al que no lo hacía. Rocha, Marinho, Jidovtseff, Silva \& Costa (2016), en una muestra de portugueses de cuatro años, comprobaron que los que realizaban dos sesiones por semana de fútbol y natación extracurricular durante 45 minutos/sesión mejoraron más sus puntuaciones en el coeficiente motor grueso y en las escalas del test (HL y HCO) en comparación con los del grupo control tras cinco y diez meses de práctica deportiva. De forma similar en el contexto español, Delgado-Lobete y Montes-Montes (2017) encontraron que las niñas y niños asturianos (4.1 años) que practicaban deporte extraescolar regularmente mostraron mayor puntuación en la Escala Observacional del Desarrollo que aquellos que no lo hacían. Además de las asociaciones encontradas con las HMB y otras áreas del desarrollo, Delgado-Lobete, Santos-delRiego, Pértega-Díaz y Montes-Montes (2019) comprobaron que la práctica de actividad física extracurricular durante tres horas o más a la semana redujo a la mitad el riesgo de padecer el trastorno del desarrollo de la coordinación.

A partir de todas las evidencias anteriores, se deduce que la práctica de actividades físico-deportivas extracurriculares puede suponer una oportunidad y una alternativa para mejorar la competencia motriz en la etapa preescolar, en tanto que supone un contexto que estimula la maduración de los patrones motores y el desarrollo de otras capacidades que apoyan el de las HMB, como las perceptivomotrices, las coordinativas y las de equilibrio. Además, la práctica deportiva permite introducir habilidades que no surgen de forma natural durante el juego libre, promoviendo así mayores niveles de logro (Stocke et al., 2014).

No obstante, no todos los contextos de práctica deportiva son válidos para fomentar la competencia en las HMB de los preescolares. Así, Šalaj et al. (2016), con una muestra de croatas de cuatro a seis años, comprobó que la competencia mejoró en el grupo que realizó un programa multideportivo (fútbol, baloncesto, voleibol, balonmano, bádminton, tenis, atletismo, gimnasia, orientación, artes marciales y bolos) pero no lo hizo en otros dos dedicados a la gimnasia rítmica y al fútbol exclusivamente. Según los investigadores, el programa multideportivo fomentó una experimentación más rica, variada y diversa que incrementó los niveles de competen- 
cia, mientras que los instructores de gimnasia rítmica y fútbol priorizaron las habilidades específicas de cada modalidad deportiva, limitando el desarrollo de todas las HMB y generando una especialización temprana. En consecuencia, teniendo en cuenta la relevancia de las HMB para sustentar las respuestas motrices más especializadas (Gallahue, Ozmun \& Goodway, 2011) y generar un estilo de vida físicamente activo y saludable (Lubans et al., 2010; Stooden et al., 2008), las actividades físico-deportivas en la etapa preescolar deberían evitar el uso excesivo de las habilidades específicas de cada deporte. Contrariamente, deberían proponer estímulos variados y de complejidad creciente, que supongan un desafío a resolver empleando HMB de distinta naturaleza y requiriendo la adaptación a un entorno cambiante.

El hecho de que las puntuaciones en las HCO fueran significativamente inferiores a las de locomoción en el grupo que no realizó deporte extraescolar, confirma la aparición más frecuente y natural de la carrera, los saltos y demás HL en los juegos de los infantes (Rocha et al., 2016). Mientras que las puntuaciones semejantes para el grupo que sí practicó deporte extracurricular podría deberse a la participación en actividades tipo fútbol, baloncesto, balonmano u otros similares, que demandan constantemente el manejo de móviles. En ambos casos se pone de manifiesto la estrecha relación entre la competencia motriz de los preescolares y el tipo de estímulos que reciben.

A pesar de los mejores resultados del grupo que realizó actividades físico-deportivas fuera del horario escolar, los percentiles que consiguieron solo fueron del 52 en el coeficiente motor grueso, del 53 en la escala de HL y del 49 en la de HCO, es decir, los preescolares de la muestra necesitaron de esas dos horas extras a la semana para conseguir la competencia motriz correspondiente con su edad teórica (Ulrich, 2000). Si a esta realidad le añadimos los percentiles alcanzados por el grupo que no realizó práctica deportiva extracurricular (coeficiente motor grueso = 17; HL = 25; HCO $=18$ ) se puede deducir que la educación motriz que reciben los niños y niñas en el ámbito formal podría no ser suficiente para promover la competencia en las HMB. Este déficit podría explicarse parcialmente a partir de los datos señalados por Pons \& Arufe (2016), quienes detectaron que el 18\% de las escuelas infantiles gallegas no incluían sesiones de educación física y que el 30\% de las clases de motricidad se desarrollaban en espacios no diseñados para la práctica de actividad física (aulas de clase, usos múltiples, salón de actos, etc.). Otra posible causa a tener en cuenta sería el tiempo dedicado a la actividad física en las escuelas. En este sentido Pons \& Arufe (2016) estimaron un promedio de 63 minutos a la semana de actividades motrices dirigidas repartidas entre una y tres sesiones. Si a este dato le añadimos los 150 minutos de recreo a la semana, obtendríamos el tiempo semanal máximo que los preescolares podrían estar físicamente activos en el marco escolar. Teniendo en cuenta que los infantes pasan 25 horas a la semana en las escuelas infantiles y que el 52\% de la población española menor de 14 años tiene hábitos sedentarios en su tiempo de ocio (INE, 2018), parece que los 183 minutos resultantes de actividad física por semana serían insuficientes, especialmente si consideramos la recomendación de 180 minutos/día a diferentes intensidades para la población de cinco años (Piercy et al., 2018). A las causas anteriores cabría añadir otra relacionada con la figura del docente, dado que se ha encontrado que los maestros de educación infantil no tienen la capacidad necesaria para implementar programas de educación física, dar las instrucciones y motivar al alumnado con el objetivo de mejorar los patrones motores (Wick et al., 2017). En el contexto gallego, las sesiones de motricidad son dirigidas por el profesorado de educación infantil o por los especialistas de educación física de primaria. En el caso de los primeros, la formación en el área de la didáctica de la expresión corporal es muy reducida y, los segundos, no suelen tener formación relacionada con las características y necesidades de los niños y niñas de preescolar. En síntesis, existen varios factores en el contexto de la educación infantil gallega que podrían mejorarse para fomentar el desarrollo motor y los niveles de competencia en las HMB. El tiempo dedicado a las actividades motrices, las características de los espacios y la formación de los profesionales son aspectos clave relacionados con la calidad de práctica motriz que deben contemplarse para poder satisfacer las necesidades del alumnado.

Otro hallazgo de este estudio fue la correlación del IMC con el coeficiente motor grueso, la escala de HL y de HCO, así como con las habilidades de galopar, saltar un obstáculo y chutar. Este hecho se tradujo en que el porcentaje de preescolares con sobrepeso u obesidad fuera significativamente mayor en el grupo que no realizó práctica deportiva (35\%) que en el que sí la hizo (13\%). La prevalencia de sobrepeso y obesidad infantil de los preescolares que no hicieron deporte coincidió con la estimación realizada por la OMS (2019) para la región europea que, además, señala que el $60 \%$ de los niños con sobrepeso u obesidad seguirán siéndolo en la adolescencia y en la etapa adulta. En el mismo sentido, Lin, Cherng \& Chen (2017), con una muestra de taiwaneses de 3 a 6 años, encontraron que los infantes con problemas motores (5 de percentil) y en riesgo de sufrirlos (5-15 de percentil) presentaron mayor porcentaje de obesidad (17-25\%) que los niños con un percentil superior a 15 (12\%). Asimismo, los preescolares con sobrepeso u obesidad invirtieron menos tiempo en realizar actividad física.

A largo plazo, los estudios que han analizado la relación entre la competencia motriz, el IMC y el sedentarismo en otras etapas educativas obtuvieron resultados similares a los expuestos en la de preescolar. Lubans et al. (2010) encontraron que el IMC y la competencia motriz se asociaron negativamente en una revisión sistemática en niños y jóvenes de tres a 18 años. Adank, Van Kann, Hoeboer, de Vries, Kremers \& Vos (2018) encontraron que los escolares con mayor competencia motriz eran menos sedentarios y dedicaban más tiempo a realizar actividad física de intensidad moderada y vigorosa. Burns, Kim, Byun \& Brusseau (2019) obtuvieron que los escolares de primaria que realizaban más actividad física moderada e intensa alcanzaron mejores resultados en el coeficiente motor grueso y en la escala de HCO que los que invertían más tiempo en comportamientos sedentarios o en actividad física de intensidad ligera. Todas estas evidencias confirman parte de la teoría de Stodden et al. (2008), estableciendo la competencia en las HMB durante la primera infancia como un determinante de la composición corporal (Barnett et al., 2016), del nivel de condición física (Catuzzo et al., 2016), de la adherencia a la práctica de actividad física 
(Logan et al., 2015) y de la propia percepción de la competencia (LeGear et al., 2102) a lo largo de toda la vida (Lubans et al., 2010).

Respecto las limitaciones del estudio, cabría señalar que no se tuvieron en cuenta las disciplinas deportivas en las que participaron los preescolares del grupo (A). Asimismo, se desconocían los enfoques metodológicos con los que se abordó la iniciación deportiva en cada caso. Teniendo en cuenta que algunos contextos tienden a la especialización temprana (Šalaj et al., 2016), sería interesante tener presente esta cuestión en futuros trabajos y discriminar tanto la influencia de la modalidad deportiva como de la orientación metodológica (resultado versus proceso) en la competencia de las HMB. De la misma forma, tampoco se tuvieron en cuenta las variables contextuales en la que los preescolares recibieron las clases de motricidad en las escuelas infantiles, lo que podría influir en los resultados si consideramos los obtenidos en otros estudios sobre los docentes (Adamo, et al., 2016) o la configuración del espacio (Barnett et al., 2013). Otra cuestión que añadir sería las características de la muestra, que no permiten extrapolar los resultados al conjunto de preescolares españoles debido al número de participantes y a la región de procedencia. Por último, el diseño de la investigación no permite conocer los efectos a largo plazo de las actividades físico-deportivas extracurriculares en la competencia motriz, aspecto de especial importancia para comprobar la relevancia de las HMB en los estilos de vida físicamente activos y saludables.

\section{Conclusiones}

A partir del objetivo de la investigación y de los resultados obtenidos se concluye que, en la muestra analizada, la competencia en las HMB se asoció positivamente con la práctica de dos horas/semana de actividades físico-deportivas extracurriculares durante un mínimo de seis meses. Asimismo, los preescolares menos competentes a nivel motor presentaron mayores índices de sobrepeso y obesidad. Dada la importancia de lograr una buena competencia en las HMB como precursora de un estilo de vida saludable se hace necesario conocer en profundidad los contextos en los que se desarrolla la educación motriz en las escuelas infantiles con nuevos estudios, así como promover su desarrollo tanto desde la educación formal como desde las actividades físico-deportivas extracurriculares.

\section{Referencias}

Adamo, K., Wilson, S., Harvey,A. L., Grattan, K. P., Naylor, P.-J., Temple, V.A., \& Goldfield, G. S. (2016). Does Intervening in Childcare Settings Impact Fundamental Movement Skill Development? Medicine and Science in Sports and Exercise, 48(5), 926-932. doi: 10.1249/MSS.0000000000000838.

Adank, A. M., Van Kann, D. H. H., Hoeboer, J.A. A., de Vries, S. I., Kremers, S. P. J., \& Vos, S. B. (2018). Investigating motor competence in association with sedentary behavior and physical activity in 7-to 11-year-old children. International Journal of Environmental Research and Public Health, 15(11), 2470. https://doi.org/10.3390/ijerph15112470.

Bardid, F., Lenoir, M., Huyben, F., De Martalaer, K., Seguers, J.,
Goodway, J. D., \& Deconick, F. J.(2017). The effectiveness of a community-based fundamental motor skill intervention in children aged 3-8years: Results of the «Multimove for Kids» project. Journal of Science and Medicine in Sport, 20(2), 184-189. doi:10.1016/j.jsams.2016.07.005.

Barnett, L., Hinkley, T., Okely, A. D., \& Salmon, J. (2013). Child, family and environmental correlates of children's motor skill proficiency. Journal os Science and Medicine in Sport, 16(4), 332-336. doi: 10.1016/j.jsams.2012.08.011.

Barnett,L. M., Lai, S. K., Veldman, S.L.C., Hardy,L.L., Cliff, D.P., Morgan, P. J., Zask, A., Lubans, D. R., Shultz, S. P., Ridgers, N. D., Rush, E., Brown, H. L., \& Okely,A. D. (2016). Correlates of Gross Motor Competence in Children and Adolescents: A Systematic Review and Meta-Analysis. Sports Medicine, 46(11), 1663-1688.https://doi.org/10.1007/s40279-016-0495$\mathrm{z}$

Batalla, A. (2000). Habilidades Motrices. Barcelona: Inde.

Becker, D. R., McClelland, M. M., Loprinzi, P., \& Trost, S. G. (2014). Physical activity, self-regulation, and early academic achievement in preschool children. Early Education and Development, 25(1), 56-70. https://doi.org/10.1080/ 10409289.2013.780505.

Bekhechi, A. K., \& Khiat, B. (2019). Impact of regular physical activity and sports on school performance among girls and boys aged between 6 and 10 years. Retos: Nuevas tendencias en Educación Física, Deporte y Recreación, 36, 397401. https://recyt.fecyt.es/index.php/retos/article/view/67113.

Burns, R. D., Kim, Y., Byun, W., \& Brusseau, T. A. (2019). Associations of school day sedentary behavior and physical activity with gross motor skills: use of compositional data analysis. Journal of Physical Activity and Health, 16(10), 811-817.https://doi.org/10.1123/jpah.2018-0549.

Callcott, D., Hammond, L., \& Hill, S. (2015). The Synergistic Effect of Teaching a Combined Explicit Movement and Phonological Awareness Program to Preschool Aged Students. Early Childhood Education Program, 43(3), 201-211. https:// doi.org/10.1007/s10643-014-0652-7.

Catuzzo, M. T., Dos Santo Henrique, N., Ré,A. H., de Oliveira, I. S., Melo, B. M., de Sousa Moura, M., de Araújo, R. C., \& Stodden, D. (2016). Motor competence and health related physical fitness in youth: A systematic review. Journal of Science and Medicine in Sport, 19(2), 123-129. https:// doi.org/10.1016/j.jsams.2014.12.004.

Cools, W., De Martelaer, K., Samaey, C, \& Andries, C. (2011). Fundamental movement skill performance of preschool children in relation to family context. Journal of Sports Sciences, 29(7),649-660. doi: 10.1080/02640414.2010.551540.

Delgado-Lobete, L., \& Montes-Montes, R. (2015). Práctica de actividad física extracurricular y preferencias deportivas en niños preescolares españoles e inmigrantes: Un estudio piloto. Revista Terapia Ocupacional Galicia, 12(22), 1-12.

Delgado-Lobete, L., \& Montes-Montes, R. (2017). Relación entre el desarrollo psicomotor y la práctica de deporte extracurricular en niños/as de tres a seis años. Sportis. Scientific Journal of School Sport, Physical Education and Psychomotricity, 3(1), 83-99.

Delgado-Lobete, L., Santos-del-Riego, S., Pértega-Díaz, S., \& Montes-Montes, R. (2019). Prevalence of suspected developmental coordination disorder and associated factors in Spanish classrooms. Research in Developmental 
Disabilities, 86, 31-40. https://doi.org/10.1016/ j.ridd.2019.01.004.

de Onis, M., \& Lobstein, T. (2010). Defining obesity risk status in the general childhood population: Which cut-offs should we use?. International Journal of Pediatric Obesity, 5(6), 458-460. doi: $10.3109 / 17477161003615583$.

Gallahue, D. L., \& Donelly, F. C. (2003). Developmental Physical Education for All Children. Champaign: Human Kinetics.

Gallahue, D. L., Ozmun, J., \& Goodway, J.(2011). Understanding motor development: infants, children, adolescents. Boston: McGraw-Hill.

González, Z., Cecchini, J.A., López, J., \& Riaño, C. (2009). Disponibilidad de las Habilidades Motrices de 4 a 14 años. Aplicabilidad del test de Desarrollo Motor Grueso de Ulrich. Aula abierta, 27(2), 19-28.

Instituto Nacional de Estadística [INE]. (2018). Encuesta Nacional de Salud. Recuperado de: http://www.ine.es/jaxi/ Tabla.htm?path=/t15/p419/a2017/p03/l0/\& file=04006.px\&L=0(marzo, 2019).

LeGear, M., Greyling, L., Sloan, E., Bell, R. I.,Willianm, B.L., Naylor, P. J., \& Temple, V.A. (2012).A window of opportunity? Motor skills and perceptions of competence of children in kindergarten. International Journal of Behavioral Nutrition and PhysicalActivity, 9(29). doi: 10.1186/1479-5868-9-29.

Lin, L. Y., Cherng, R. J., \& Chen, Y. J. (2017). Relationshipbetween time use in physical activity and gross motor performance of preschool children. Australian occupational therapy journal,64(1), 49-57. doi:10.1111/1440-1630.12318.

Logan, S. W., Webster, E. K., Getchell, N., Pfeiffer, K. A., \& Robinson, L. E. (2105). Relationship between fundamental motor skill competence and physical activity during childhood and adolescence: Asystematic review. Kinesiology Review, 4,416-426. https://doi.org/10.1123/kr.2013-0012.

Lubans, D. R., Morgan, P. J., Cliff, D. P., Barnett, L. M., \& Okely,A. (2010). Fundamental movements skills in children and adolescents: Review of associated health benefits. Sports Medicine, 40(12), 1019-1035. doi: 10.2165/11536850000000000-00000.

Malina, R. M., Bouchard, C., \& Bar-Or, O. (2004). Growth, Maturation and Physical Activity. Champaign: Human Kinetics.

Malina, R. M.(2009). Children and adolescents in the sport culture: the overwhelming majority to the select few. Journal of exercise science \& fitness, 7(2), 1-10. https://doi.org/10.1016/ S1728-869X(09)60017-4.

Padial-Ruz, R., Ibáñez-Granados, D., Fernández, H., \& UbagoJiménez, J. L. (2019). Flamenco dance project: motor and emotional development in early childhood education. Retos: Nuevas tendencias en Educación Física, Deporte y Recreación, 35, 396-401. https://recyt.fecyt.es/index.php/retos/ article/view/63292.

Piercy, K. L., Troiano, R. P., Ballard, R. M., Carlson, S.A., Fulton, J. E., Galuska, D. A., ... \& Olson, R. D. (2018). The physical activity guidelines for Americans. Jama, 320(19), 2020-2028. doi:10.1001/jama.2018.14854.

Pons, R., \& Arufe, V.(2016). Análisis descriptivo de las sesiones e instalaciones de psicomotricidad en el aula de Educación Infantil. Sportis. Scientific Journal of School Sport, Physical Education and Psychomotricity, 2(1), 125-146. https:// doi.org/10.17979/sportis.2016.2.1.1445.

Queiroz,D.D. R., Ré,A.H. N., Henrique, R.D. S., Moura, M.D.S., \& Cattuzzo, M. T. (2014). Participation in sports practice and motor competence in preschoolers. Motriz: Revista de Educação Física, 20(1), 26-32. http://dx.doi.org/10.1590/ S1980-65742014000100004.

Robinson, L. E., Palmer, K. K., \& Bub, K. L. (2016). Effect of the Children's Health Activity Motor Program on Motor Skills and Self-Regulation in Head Start Preschoolers: An Efficacy Trial. Frontiers in Public Health, 4(173), 1-9. doi: 10.3389/ fpubh.2016.00173.

Rocha, H.A., Marinho, D. A., Jidovtseff, B., Silva, A. J., \& Costa, A. M.(2016). Influence of regular soccer or swimming practice on gross motor development in childhood. Motricidade, 12(4), 33-43. https://doi.org/10.6063/motricidade.7477.

Šalaj, S., Krmpotiæ, M., \& Stamenkoviæ, I. (2016). Are specific programs a threat to overall motor development of preschool children?. Kinesiologia slovenica, 22(1), 47.

Spessato, B., Gabbard, C., Valentini, N., \& Rudisill, M. (2012). Gender differences in Brazilian children's fundamental movement skill performance. Early Child Development and Care, 183(7), 916-923. https://doi.org/10.1080/ 03004430.2012.689761.

Stocke, M., Oliveira, B., \& Cristina, N. (2014). Guided play and free play in an enriched environment: Impact on motor development.Motriz,20(2), 177-185.http://dx.doi.org/10.1590/ S1980-65742014000200007.

Stodden, D. F., Goodway, J. D., Langendorfer, S. J., Roberton, M. A., Rudisill, M. E., García, C., \& García, L. E. (2008). A Developmental Perspective on the Role of Motor Skill Competence in Physical Activity: An Emergent Relationship. Quest, 60(2), 290-306. https://doi.org/10.1080/ 00336297.2008 .10483582$.

Ureña, N., Ureña, F., \&Alarcón, F. (2008) Una propuesta de evaluación para las habilidades motrices básicas en Educación Primaria a través de un juego popular: la oca. Retos: Nuevas tendencias en Educación Física, Deporte y Recreación, 14,35-42.https://recyt.fecyt.es/index.php/retos/article/view/ 35008.

Veldman, S., Palmer, K. K., Okely,A. D., \& Robinson,L.E.(2017). Promoting ball skills in preschool-age girls. Journal of Science and Medicine in Sport, 20(1), 50-54. doi: 10.1016/ j.jsams.2016.04.009.

Wick, K., Leeger-Aschmann, C. S., Monn, N. D., Radtke, T., Ott, L. V., Rebholz, C. E., \& Munsch, S. (2017). Interventions to promote fundamental movement skills in childcare and kindergarten: a systematic review and meta-analysis. Sports Medicine, 47(10), 2045-2068. doi: 10.1007/s40279-017-07231.

Wickel, E. E., \& Eisenmann, J. C. (2007). Contribution of youth sport to total daily physical activity among 6-to 12-yr-old boys. Medicine and science in sports and exercise, 39(9), 1493-500. doi: 10.1249/mss.0b013e318093f56a.

Wu, M., Lian, X., Lu, S., \& Wang, Z. (2017). Infant motor and cognitive abilities and subsequent executive function. Infant Behavior and Development, 49, 204-213. doi: 10.1016/ j.infbeh.2017.09.005. 